\title{
Dietary sulfate-driven and gut dysbiosis-triggered breast cancer-related gene upregulation
}

\author{
CHEN YanPing† LIAO Tao† TAN LiLi CHEN DongMei XU Qin Song JianPing* ZHEN QingPing* \\ Tropical Medicine Institute, Guangzhou University of Chinese Medicine, Guangzhou 510405 \\ † Equal contributors \\ *Correspondence, E-mail: qpzeng@gzucm.edu.cn; songjp@gzucm.edu.cn
}

\begin{abstract}
By gut microbiota metagenomic analysis, we found that the abundance of sulfatase-secreting bacteria (SSB) in the gut of mice fed chondroitin sulfate (CS) increases with significant individual difference. The fluctuation of lipopolysaccharide (LPS) and pro-inflammatory indicators with significant individual and tissue variations was also observed. After mice were fed mixed with CS or injected separately with LPS, the breast cancer-related transcriptional factor genes, $B C L 11 A$ and $R U N X 1$, were upregulated, whereas the tumor suppressor gene, TP53BP1, were downregulated. Further, the mammary myopithelium marker CK5/6, the mammary hyperplasia marker Ki-67, and other tumor markers were also upregulated. While the exogenous estradiol does not induce the expression of BCL11A, RUNX1, and TP53BP1, the estrogen receptor (ER) agonist Fulvestrant that mimics estradiol action not only elevates estradiol concentrations, but also upregulates tumor marker expression levels, revealing that ER inflammatory inactivation and hyperestrogenemia induction might be the etiological cues of breast cancer origin. This study has preliminarily established a possible correlation of gut microbiota dysbiosis and chronic low-grade inflammation with the early-phase onset of breast cancer in mice. The statistical insignificance of test data was attributed to the individual difference of gut microbiota compositions, which determining the individual and tissue variations of systemic inflammation.
\end{abstract}

Keywords: Breast cancer, etiological hypothesis, gut microbiota, expression profile, chronic low-grade inflammation

The statistical data in 2015 have indicated that the global incidence rate of breast cancer was up to $12 \%$ among women ${ }^{[1]}$. In all patients, only $2-3 \%$ cases are belonging to familiar early-onset breast cancer, which are resulted from $B R C A 1$ or $B R C A 2$ gene mutation, and others are non-genetically late-onset breast cancer ${ }^{[2]}$, whose etiological reasons remain unknown. Nevertheless, accumulating evidence shows that hyperestrogenemia has been considered as the critical inducer of breast cancer ${ }^{[3]}$, in a possible mechanism underlying that 
estrogens stimulate estrogen receptors (ERs) and further activate the downstream cell division-promoting transcription ${ }^{[4]}$. For the source of high-level estrogens, the current explanation is that colon cell-secreted $\beta$-glucuronidase converts estrogens from the bound-types to the free types, enabling re-adsorption via the enterohepatic circulation ${ }^{[5]}$.

On the other hand, some evidence indicates that the unhealthy lifestyles, including a high-fat diet ${ }^{[6]} 、$ smoking $^{[7]} 、 \operatorname{drunk}^{[8]}$, are significantly associated with breast cancer incidence, and a possible link of gut microbiota disorder to breast cancer incidence has been also concerned ${ }^{[9]}$. According to these cues, we suggest here a hypothesis of the "gut origin of breast cancer", which addresses that gut dysbiosis from opportunistic infection leads to colon mucosal damaged, bacterial endotoxin leaked into blood circulation, and systemic inflammation induced ${ }^{[10]}$. Along with the inflammatory lesion and functional inactivation of ERs, estrogen levels should be complementarily elevated, and oncogenes accordingly upregulated or tumor suppressors downregulated, eventually inducing mammary gland hyperplasia (benign tumor) or transformation (malignant cancer).

Because animal-derived chondroitin sulfate (CS) was proven to trigger opportunistic infection from the overgrowth of sufatase-secreting bacteria (SSB) and sulfate-reducing bacteria ${ }^{[11]}$, we fed CS and SSB to female mice to mimic gut dysbiosis and induce chronic low-grade inflammation ${ }^{[12]}$. A possibility of the interaction of CS-rich meat diets with gut microbiota communities raise a breast cancer risk was evaluated by determining the expression levels of mammary tumor-related transcription factors and tumor markers. A cause of hyperestrogenemia during breast cancer incidence was proposed by comparing the expression levels of mammary tumor-related transcriptional factors upon injection of the exogenous estrogen, estradiol (ED) ${ }^{[13]}$, or the ERs agonist, Fulvestrant $(\mathrm{FS})^{[14]}$. An association of the individual gene expression difference with the inconsistent experimental repeatability was revealed by the metagenomic gut microbiome analysis ${ }^{[15]}$.

The present study preliminarily dissected the putative mechanism underlying that gut dysbiosis induces mammary tumor by eliciting systemic inflammation. On this basis, it should be realistic to improve gut ecosystems, eradicate tumorigenic cues, and decline carcinogenic risks by artificial attempts, which would create an 
innovative era for the early-phase intervention of gut dysbiosis and effective prevention of breast cancer.

\section{Materials and Methods}

\subsection{Animal group and reagent treatment}

The specific pathogen free BALB/c mice (female, 18-22 g) and Kunming mice (female, $30 \pm 2 \mathrm{~g}$ ) were provided by the experimental animal center in Guangzhou University of Chinese Medicine. The animals were allowed feeding routinely and drinking freely, and divided randomly into 6 groups with 2-4 mice in each group. (1) Group ad libitum (AL); (2) Group CS (FocusChem, Shandong, China); (3)Group CS+Bacillus cereus (BC, Huankai Microb, Guangdong, China); (4)Group CS+BC+FS(MCE); (5)Group LPS (Sigma); 6) Group low-dose ED (Alfa Aesar); (7) Group high-dose ED.

$0.25 \mathrm{ml} \mathrm{CS}(1.0 \mathrm{~g} / \mathrm{kg})$ was used to intragastrical feed group (2) mice, and repeat after $2 \mathrm{w}$; A solution containing $10^{4} \mathrm{BC}$ was used to intragastrical feed group (3) mice. On the next day, they were intragastrically fed by $0.25 \mathrm{ml} \mathrm{CS}(1.0 \mathrm{~g} / \mathrm{kg})$, and repeat after $2 \mathrm{w} ; \mathrm{FS}$ $(500 \mu \mathrm{g} / \mathrm{kg}$ ) was used to peritoneal inject group (4) mice, 1 time $/ \mathrm{d}$ for $60 \mathrm{~d}$; LPS $(0.25 \mathrm{mg} / \mathrm{kg})$ was used to peritoneal inject group (5) mice, 1 time every other day for $60 \mathrm{~d} ; 0.5 \mathrm{mg} / \mathrm{kg}$ and $4.0 \mathrm{mg} / \mathrm{kg}$ ED were used to inject group (6) and (7) mice, 3 times/w for $8 \mathrm{w}$. Fecal samples were collected from group (1) and (2) mice for gut microbiota metagenomic analysis. All mice were sacrificed and mammary, hepatic, muscular and adipose tissues were collected for determining the levels of induced gene expression.

\subsection{Quantitative polymerase chain reaction (Q-PCR)}

RNA isolation, purity determination, electrophoresis monitoring, reverse transcription, and quantification were performed obeying a

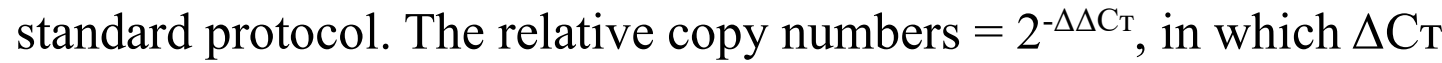
$=\mathrm{Ct}$ target gene $-\mathrm{Ct}_{\text {reference gene, }} \Delta \Delta \mathrm{CT}=\Delta \mathrm{C}$ treatment sample $-\Delta \mathrm{C}$ control sample. The primers were designed and applied to amplification under the following amplification condition: $95^{\circ} \mathrm{C}, 30 \mathrm{~s} ; 95^{\circ} \mathrm{C}, 3 \mathrm{~s}, 64^{\circ} \mathrm{C}, 34 \mathrm{~s}$, 45 cycles. The primers were listed as below: 


$\begin{array}{ll}\text { RUNX1-F } & \text { CTGCCCATCGCTTTCAAGGT } \\ \text { RUNX1-R } & \text { GCCGAGTAGTTTTCATCATTGCC } \\ \text { TP53BP1-F } & \text { ATGGACCCTACTGGAAGTCAG } \\ \text { TP53BP1-R } & \text { TTTCTTTGTGCGTCTGGAGATT } \\ \text { BCL11A-F } & \text { ACAAACGGAAACAATGCAATGG } \\ \text { BCL11A-R } & \text { TTTCATCTCGATTGGTGAAGGG } \\ \text { GAPDH-F } & \text { ACAGTCAGCCGCATCTTC } \\ \text { GAPDH-R } & \text { CTCCGACCTTCACCTTCC }\end{array}$

\subsection{Enzyme-linked immunosorbent assay (ELISA)}

Mouse LPS ELISA Kit was purchased from Jinma (Shanghai, China). Mouse TNF- $\alpha$ and TNF receptor-1 (TNFR1) ELISA Kit was purchased from Chenglin (Beijing, China).

\subsection{Metagenomic analysis}

The gut microbiota profiles in mouse fecal samples were identified by the high-throughput 16S VX sequencing-based classification procedure. The sequencing (sample preparation, DNA extraction and detection, amplicon purification, library construction and online sequencing) and data analysis (paired end-reads assembly and quality control, operational taxonomic units cluster and species annotation, alpha diversity and beta diversity) were conducted by Novogene, Beijing, China.

\subsection{Statistical analysis}

The software SPSS 22.0 was employed to analyze data, and the software GraphPad Prism 5.0 was employed to plot graphs. The Independent Simple Test was used to compare all groups, but the Kruskal-Wallis Test followed by Nemenyi test was used when the data distribution is skewed. The significance level ( $\mathrm{p}$ value) was set at $<0.05(*),<0.01(* *),<0.001(* * *)$ and $<0.0001(* * * *)$.

\section{Results and Discussion}

\subsection{CS promotes SSB overgrowth}

In the gut of healthy Chinese people, SSB such as Bacteroides thetaiotaomicron $\mathrm{J} 1$ and 82 strains, B. ovitus E3 strain, and 
Clostridium hathewayi $\mathrm{R} 4$ strain were classified ${ }^{[16]}$. Meanwhile, Akkermansia muciniphila was also confirmed to be a gut commensal species capable of secreting sulfatases ${ }^{[17]}$. After fed mice with CS, we found the increases in A. muciniphila and B.cereus abundance, in which A. muciniphila accounts for 4\% among Akkermansia, and $1.059 \%$ among all bacterial phyla; B.cereus accounts for $4.41 \%$ among Bacillus, and $1.028 \%$ among all bacterial phyla. In contrast, SSB were almost not detected in the gut of control mice, AL1 and AL2. Additionally, Escherichia coli producing LPS accounts for $1.62 \%$ in Escherichia, and $0.379 \%$ among all bacterial phyla (Figure 1).

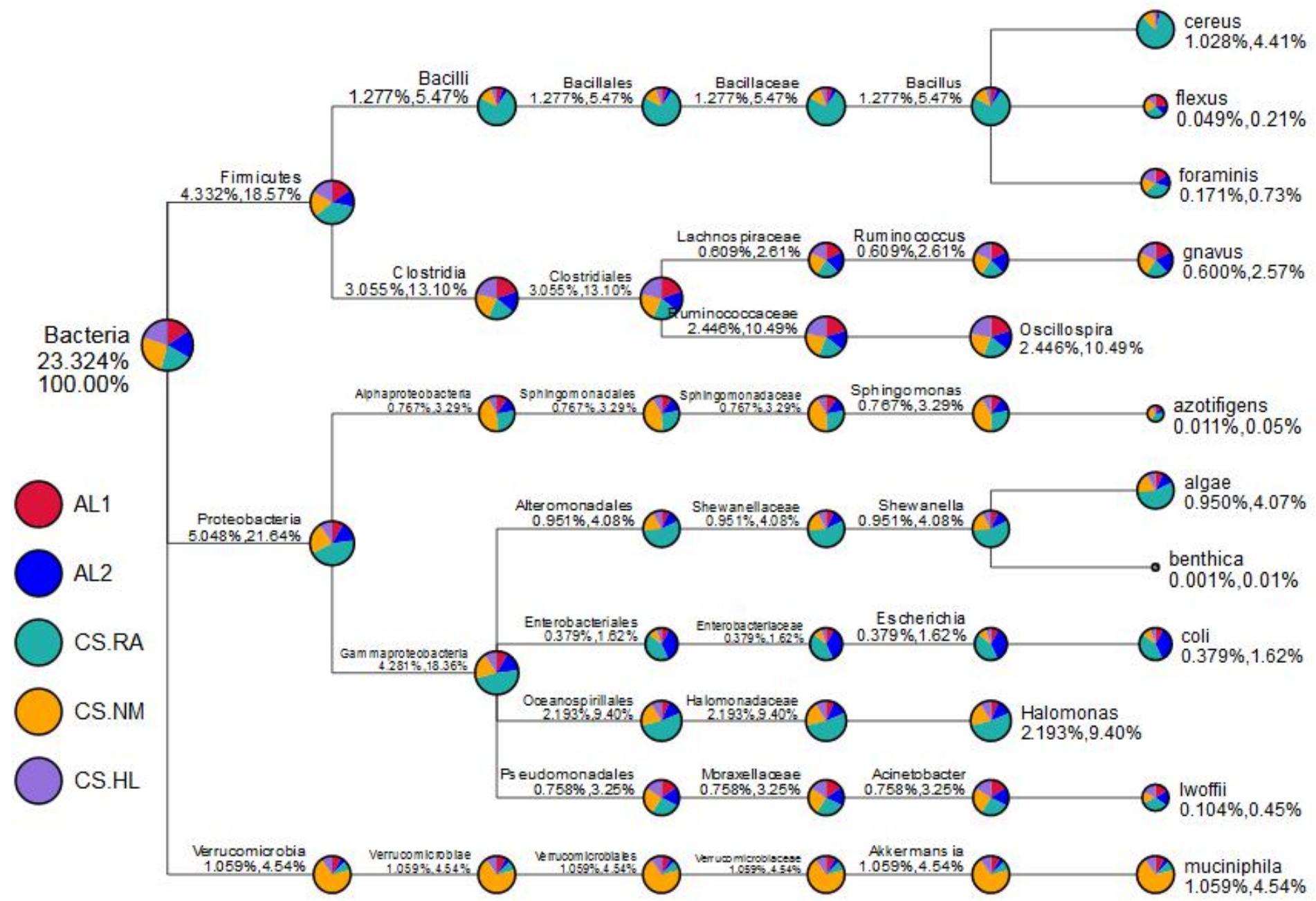

\section{Fig.1 A taxonomic tree of bacteria specific in mouse fecal}

\section{samples.}

The fan-shaped sectors in the circle represent different samples corresponding to the left legends, and the proportion of a fan-shaped sector indicates a relative abundance of the sample in the taxonomy. For two percentages listed below the taxonomic nomenclature, the former is the percentage among all species, and the letter is the percentage in the selected species. AL1, AL2: Mice fed Ad libitum chow; CS.RA, CS.NM, CS.HL: Mice fed CS. 
Although fed CS without exception, the kind and abundance of SSB in the gut of each mouse show the significant variations among individuals. The richness of $A$. muciniphila in the treated mouse, CS.NM, reaches $4.00 \%$, but that in the treated mice, CS.RA and CS.HL, is only $0.50 \%$, which is slightly higher than the average percentages, $0.30 \%$, in the control mice, AL1 and AL2. In contrast, the richness of B.cereus in the treated mouse, CS.RA, reaches $4.03 \%$, but that in the treated mice, CS.NM and CS.HL is only $0.24 \%$ and $0.65 \%$, which are equal or slightly higher than the average percentages, $0.24 \%$, in the control mice, AL1 and AL2 (Table 1).

\section{Table 1 The classification and proportion of gut SSB in mice} fed CS.

\begin{tabular}{lccccccc}
\hline \multicolumn{1}{c}{ SSB } & $\begin{array}{c}\mathrm{AL} 1 \\
(\%)\end{array}$ & $\begin{array}{c}\mathrm{AL} 2 \\
(\%)\end{array}$ & $\begin{array}{c}\overline{\mathrm{x}} \pm \mathrm{s} \\
(\%)\end{array}$ & $\begin{array}{c}\text { CS.RA } \\
(\%)\end{array}$ & $\begin{array}{c}\text { CS.NM } \\
(\%)\end{array}$ & $\begin{array}{c}\text { CS.HL } \\
(\%)\end{array}$ & $\begin{array}{c}\overline{\mathrm{x}} \pm \mathrm{S} \\
(\%)\end{array}$ \\
\hline $\begin{array}{l}\text { Akkermansia } \\
\text { municinphila }\end{array}$ & 0.40 & 0.20 & $0.30 \pm 0.14$ & 0.50 & 4.00 & 0.50 & $1.67 \pm 2.02$ \\
\hline Bacillus cereus & 0.24 & 0.24 & $0.24 \pm 0.00$ & 4.03 & 0.24 & 0.65 & $1.64 \pm 2.08$ \\
\hline
\end{tabular}

Note: AL1, AL2: Control mice fed AL chow; CS.RA, CS.NM, CS.HL: Treated mice fed CS.

These above results indicated that feeding CS to mice can change the gut microbiota composition, but not every mouse exhibits opportunistic infection by SSB, implying that the remarkable difference of SSB occurs among the gut of each individual mouse. Mounting evidence shows that the effect of CS on the female mice are more predominately than the male mice ${ }^{[18]}$. Our research has chosen the female mice for investigation, so the great individual variation of gut microbiota communities can be attributed to CS.

\subsection{SSB prompts serum LPS and pro-inflammatory mediator fluctuation}

To explore whether CS feeding would induce inflammatory responses by leaking gut microbiota debris, we determined the serum LPS and TNF- $\alpha$ levels in mice, as listed in Table 2.

\section{Table 2 The individual specificity of serum LPS and TNF- $\alpha$ in mice fed CS}

\begin{tabular}{cccccccc}
\hline Serum titer & AL1 & AL2 & $\overline{\mathrm{x}} \pm \mathrm{S}$ & CS.RA & CS.NM & CS.HL & $\overline{\mathrm{x}} \pm \mathrm{S}$ \\
\hline $\begin{array}{c}\text { LPS } \\
(\mathrm{EU} / \mathrm{L})\end{array}$ & 11.96 & 14.94 & $13.45 \pm 2.11$ & 16.18 & 16.09 & 13.29 & $15.19 \pm 1.64$ \\
\hline
\end{tabular}




\begin{tabular}{llllllll}
\hline $\begin{array}{l}\text { TNF- } \alpha \\
(\mathrm{ng} / \mathrm{L})\end{array}$ & 378.73 & 526.25 & $452.49 \pm 104.31$ & 1067.18 & 407.87 & 664.67 & $820.69 \pm 298.55$ \\
\hline
\end{tabular}

Note: AL1, AL2: Control mice fed AL chow; CS.RA, CS.NM, CS.HL: Treated mice fed CS.

From the above results, it was clear that CS feeding- induced gut opportunistic infection increases the serum LPS and TNF- $\alpha$ levels albeit no significant statistical difference between AL mice and CS mice. This phenomenon seems to be resulted from the huge individual variation of the compared raw data.

The tissue variations in LPS, TNF- $\alpha$ and TNFR1 levels were also observed. In AL1 and CS.RA mice, for example, LPS levels are declined in the hepatic, adipose and muscular tissues, whereas TNF- $\alpha$ levels are declined only in the adipose tissue, but are elevated in the adipose and muscular tissues. TNFR1 levels are elevated in the adipose and muscular tissues (Figure 2), indicating a conversion of LPS from high levels to low levels.

A

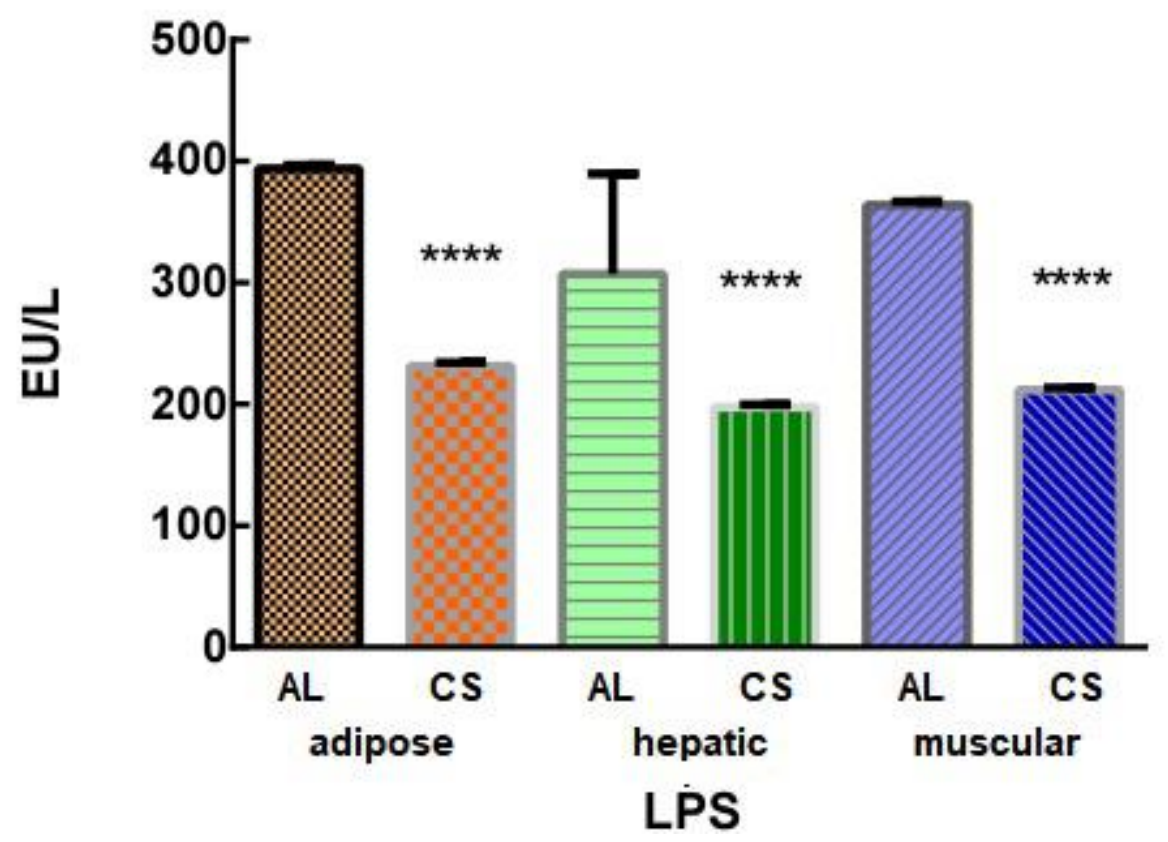


B
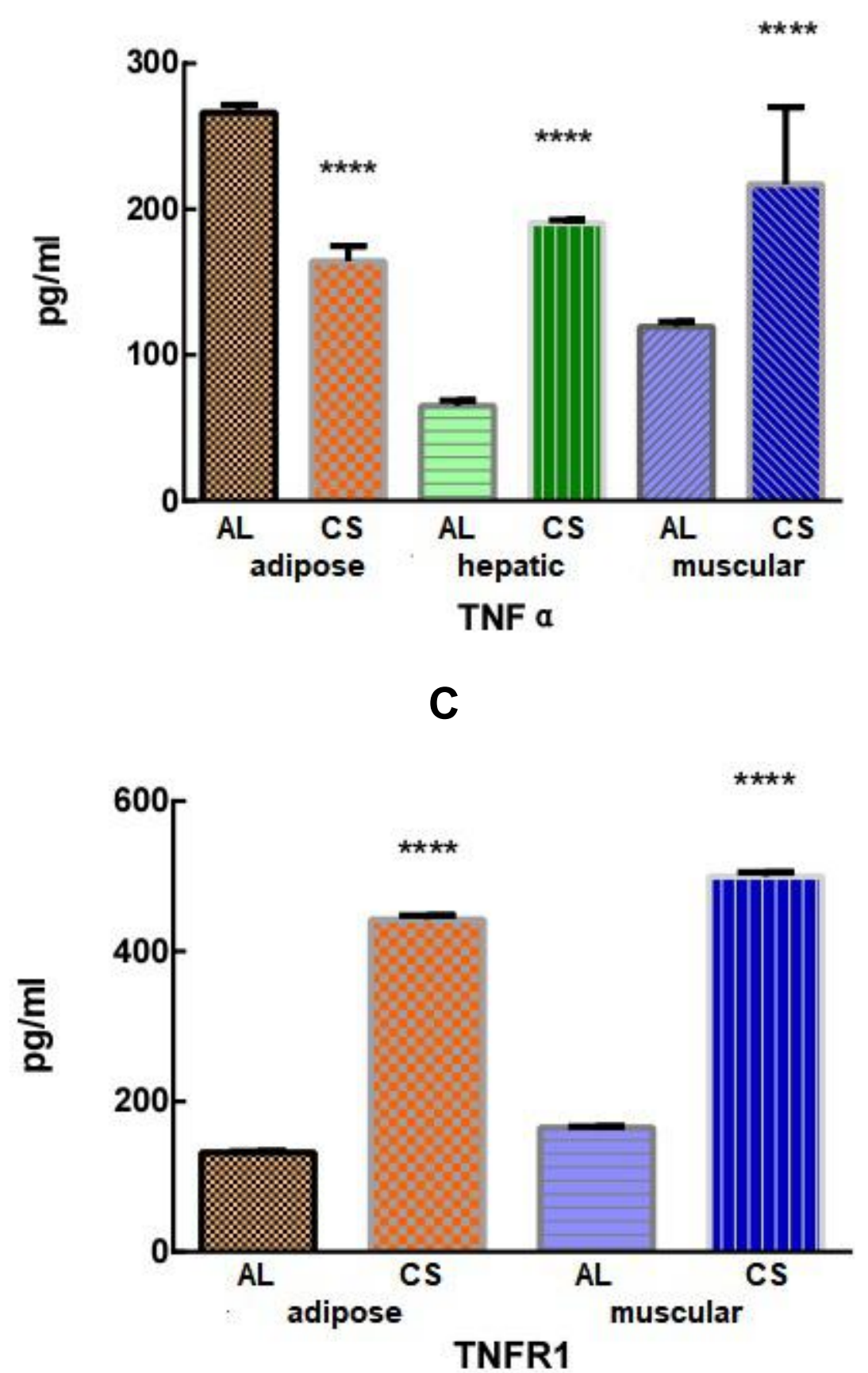

Fig.2 The tissue specificity of LPS and pro-inflammatory mediators in mice fed CS.

A. LPS levels in mouse liver, adipose, and muscular tissues; B. TNF- $\alpha$ levels in mouse liver, adipose, and muscular tissues; C. TNFR1 levels in adipose and muscular tissues. AL: Tissues from mice fed Ad libitum chow; CS: Tissues from CS-treated mice. ${ }^{* * * *} p<0.0001(n=15)$.

The tissue variations of LPS and pro-inflammatory cytokines/ receptors may be associated with the serum and tissue LPS levels and anti-LPS depleting LPS rates. Hersoug et al ${ }^{[19]}$ disclosed that 
after scavenger receptor B1 (SRB1) binds to LPS, SRB1-LPS can then be incorporated into the chylomicrons, which enhances LPS trespassing across the endothelium and entering into adipocytes. These results implied that LPS may be preferably transported into the adipose tissue, which induces anti-LPS and upregulates TNF- $\alpha$ and TNFR1, eventually eradicating LPS and downregulating TNF- $\alpha$.

\subsection{Induced expression of mouse mammary gland tumor-related transcription factor genes}

From the data of metagenomic analysis, it was predicted that the individual variation of mouse gut microbiota difference may represent the major cause of distinct inflammatory severity. So when we fed CS, we also gastrically administered BC, by which the individual variation of mouse gut microbiota difference was anticipated to be minimized. After CS+BC feeding or LPS injection, we quantified the mouse mammary mRNA levels of the mammary tumor-related transcription factor genes $B C L 11 A^{[20]}$ and $R U N X 1^{[21]}$, and the tumor suppressor TP53 binding protein-1 gene (TP53BP1) [22].

The results indicated that $B C L 11 A$ and $R U N X 1 \mathrm{mRNA}$ levels are higher in $\mathrm{CS}+\mathrm{BC}$ or LPS-treated mice than those in control mice, in which LPS-induced mRNA levels are higher than $\mathrm{CS}+\mathrm{BC}$-induced mRNA levels. As to TP53BP1 mRNA, both treatments induce the similar levels with control. Besides, $\mathrm{CS}+\mathrm{BC}+\mathrm{FS}$ significantly increase $R U N X 1 \mathrm{mRNA}$ (Figure 3 ).

A

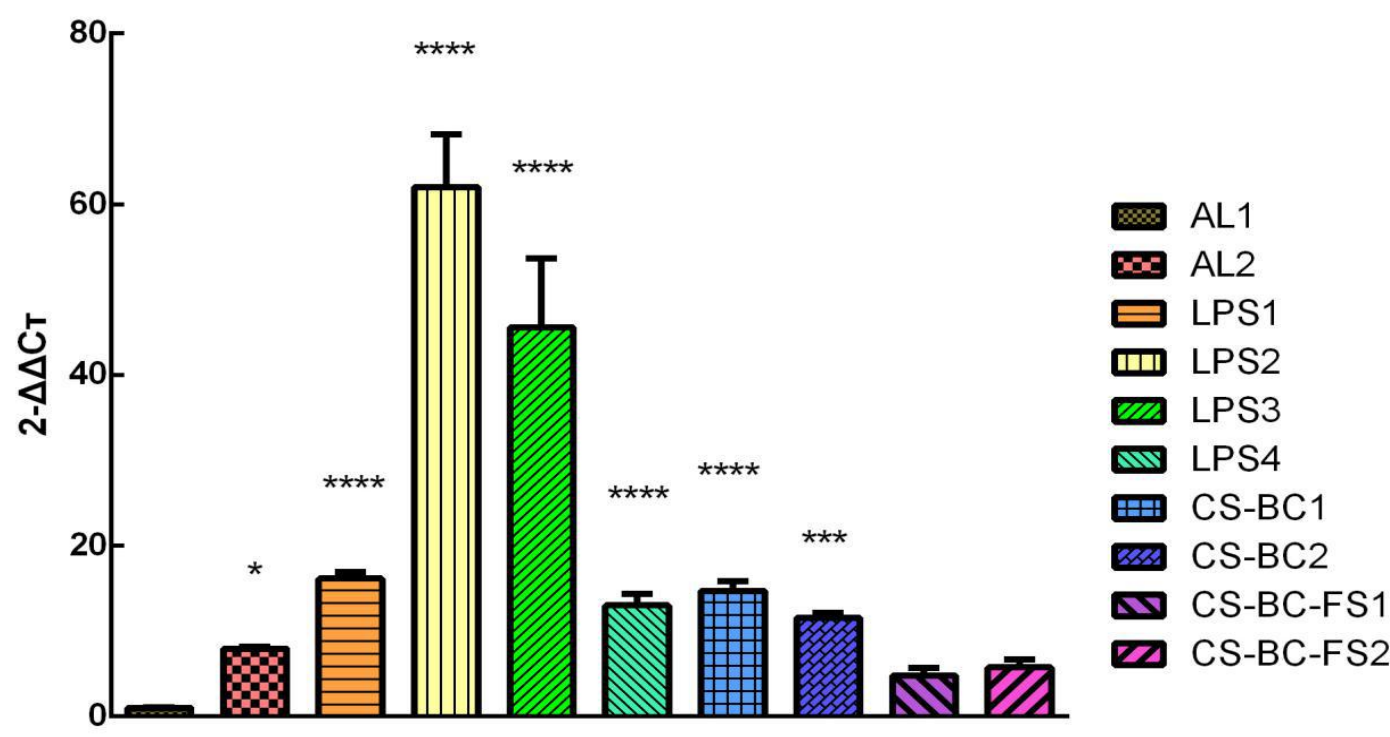

BCL11A 
B

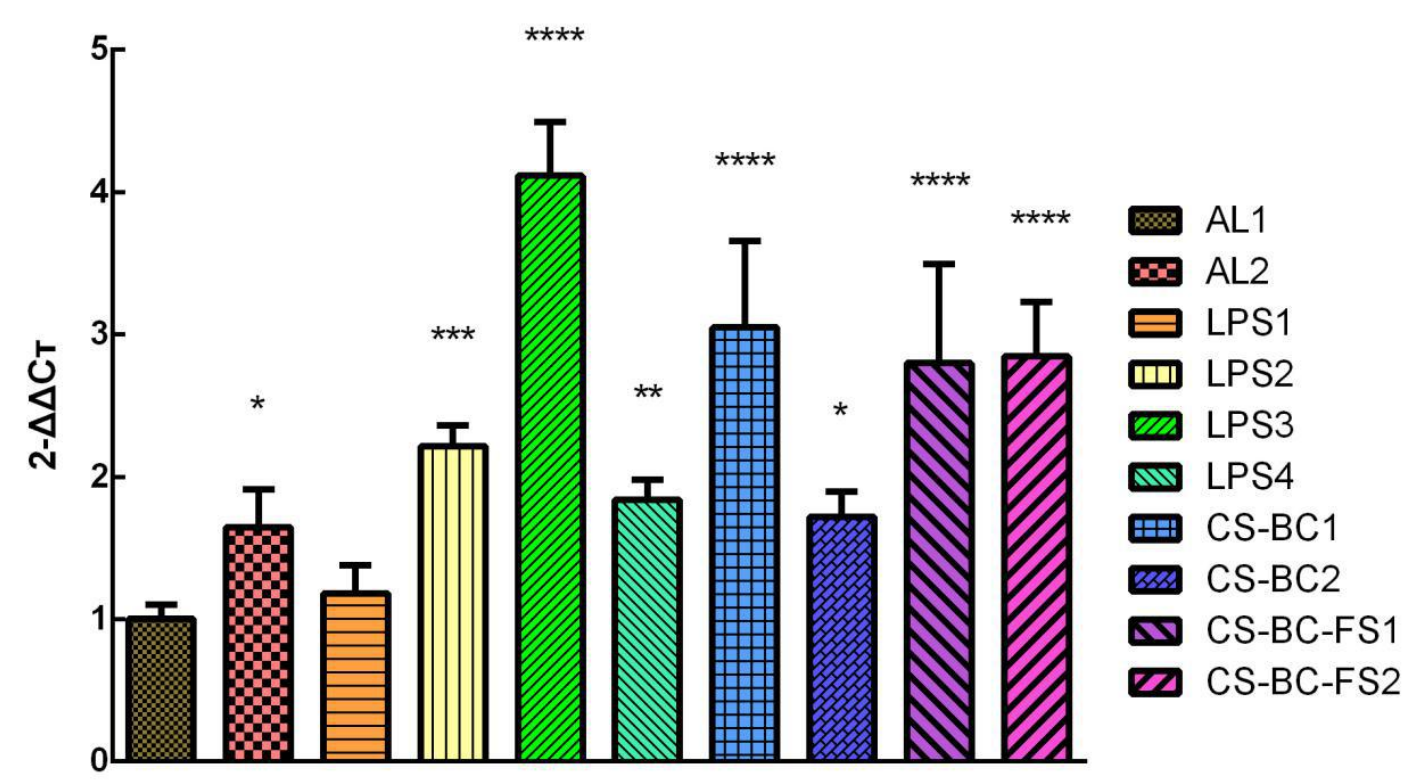

RUNX1

C

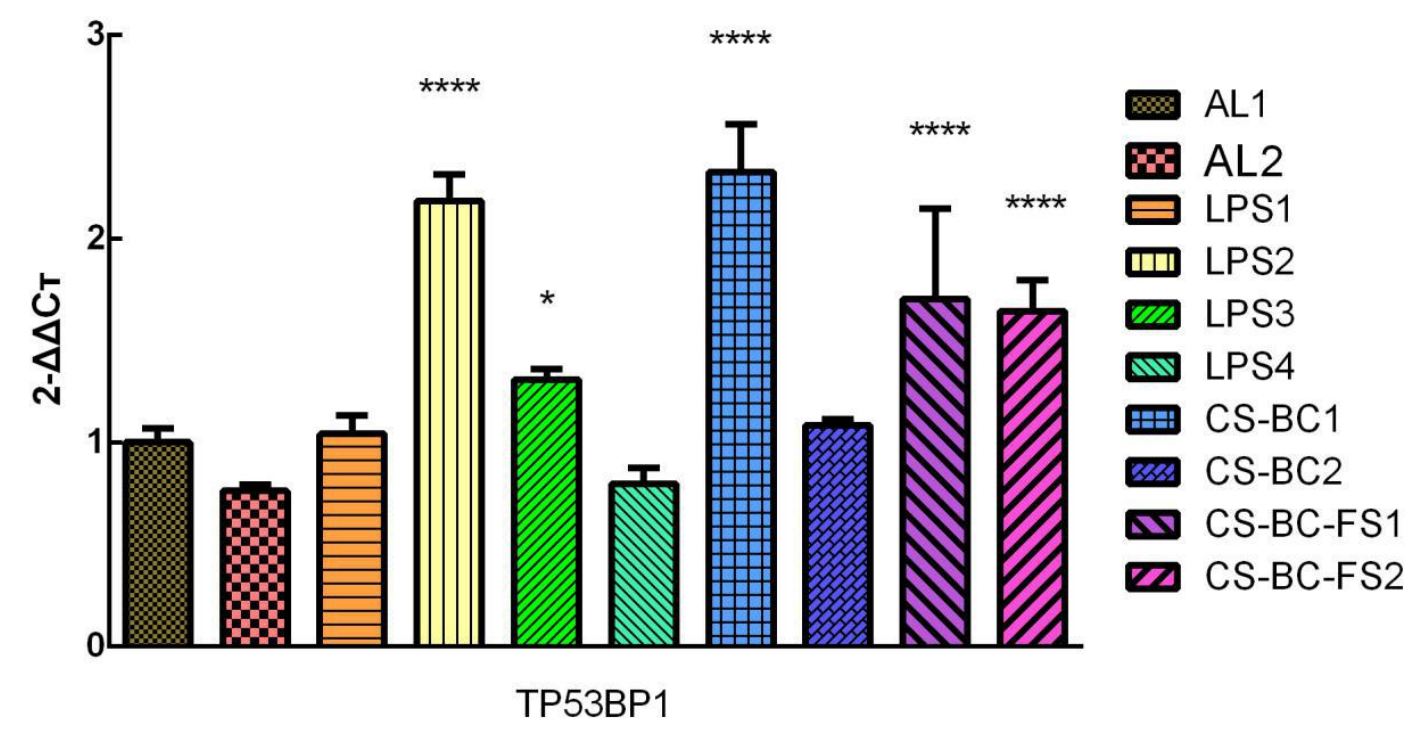

Fig. 3 The multi-factorial induction of tumor-related genes expression in mouse mammary tissues.

A. The relative $B C L 11 A$ mRNA copy numbers; B. The relative $R U N X 1$ mRNA copy numbers mRNA; C. The relative TP53BP1 mRNA copy numbers. AL: Mammary gland tissues of mice fed Ad libitum chow; LPS: LPS-treated mouse mammary gland tissues; $\mathrm{CS}+\mathrm{BC}$ : chondroitin sulfate+Bacillus cereus-treated mouse mammary gland tissues; $\mathrm{CS}+\mathrm{BC}+\mathrm{FS}$ : chondroitin sulfate + Bacillus cereus + fulvestrant-treated mouse mammary gland tissues. ${ }^{*} p<0.05 ; * * p<0.01$; $* * * p<0.001 ; * * * * p<0.0001(n=15)$. 
It could be seen from the above figure that the expression levels of tumor-related genes remain extraordinarily varied, reflecting that the attempt of uniforming the gut microbiota using $\mathrm{BC}$, which is perhaps because the activity of in vitro cultured bacteria is relatively low, and the orally administered bacteria are killed by the acidic gastric juice.

\subsection{Exogenous estradiol fails to induce $B C L 11 A 、 R U N X 1$ and TP53BP1 expression}

To evaluate the effect of ED on mammary tumor incidence, we quantified the mammary levels of BCL11A、RUNX1 and TP53BP1 mRNA in ED-injected mice. The results shown that the mammary $B C L 11 A 、 R U N X 1$ and TP53BP1 mRNA levels induced by either high-level ED $(4.0 \mathrm{mg} / \mathrm{kg})$ or low-level ED $(0.5 \mathrm{mg} / \mathrm{kg})$ are comparative to those in control mice (Figure 4).

A

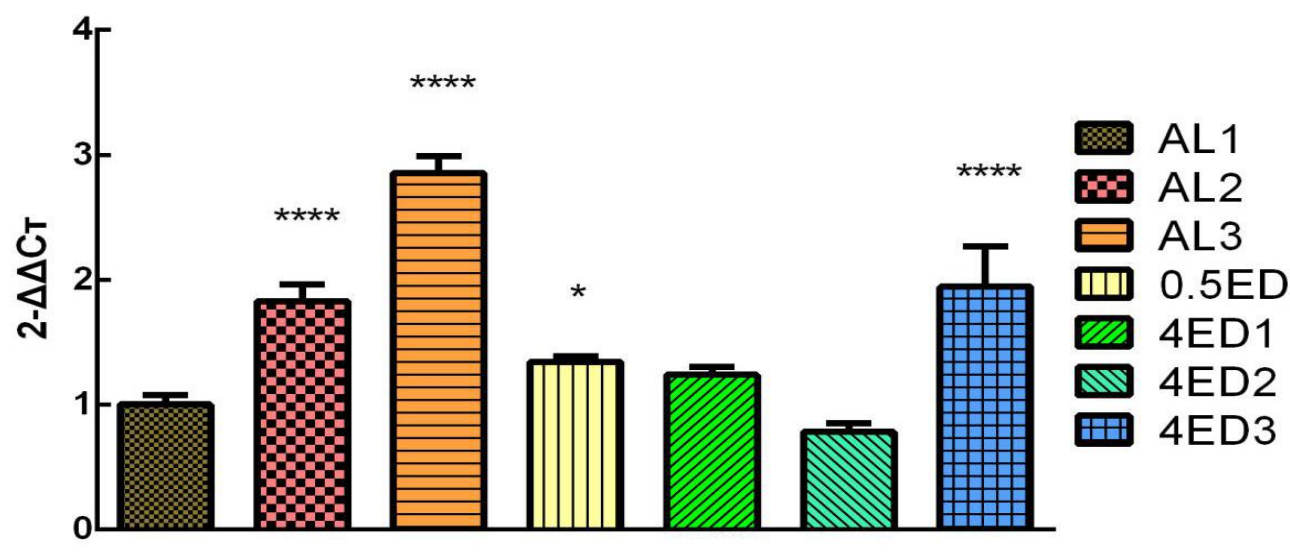

BCL11A

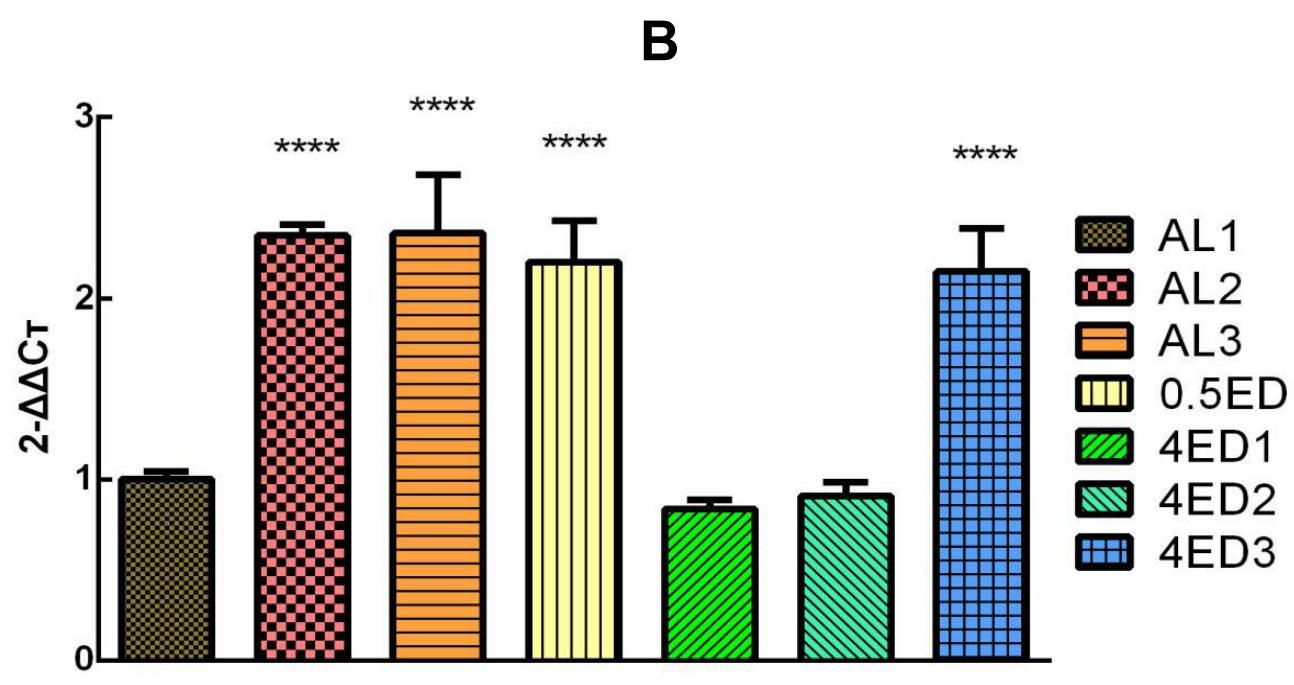

RUNX1 


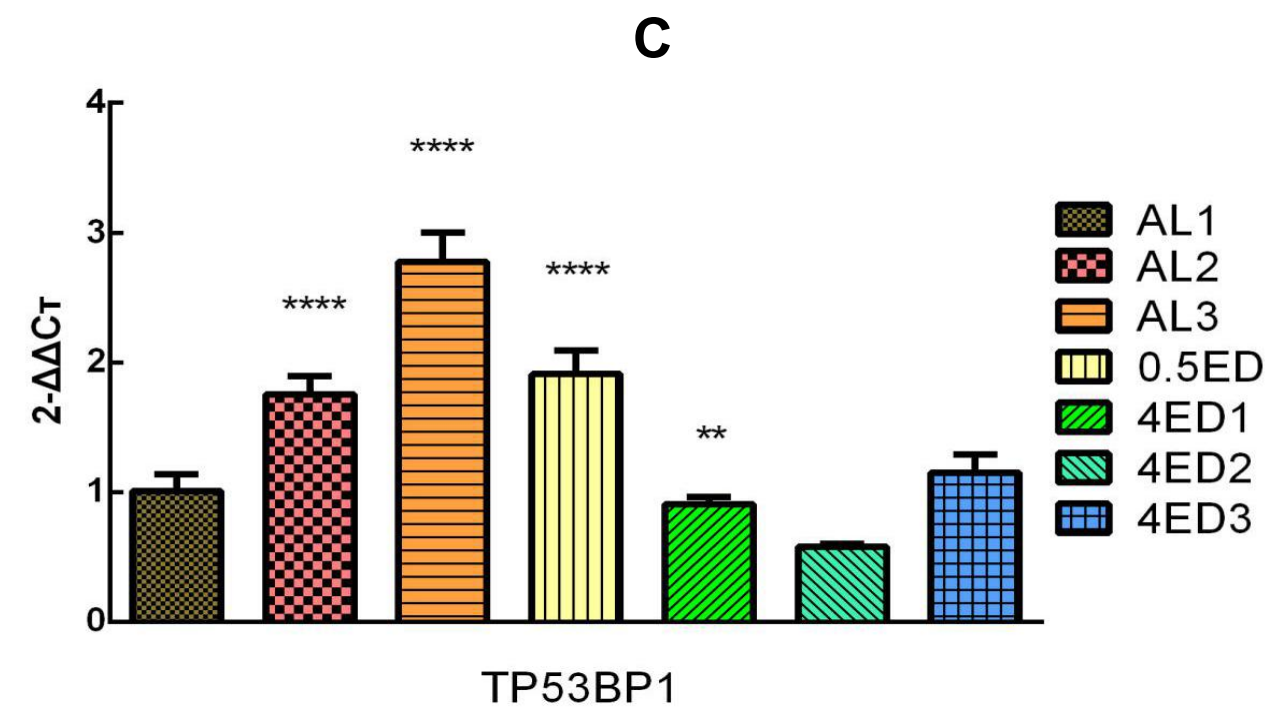

Fig. 4 The estradiol-induced expression of tumor-related genes in mouse mammary gland tissues

A. The relative $B C L 11 A$ mRNA copy numbers; $\mathrm{B}$. The relative $R U N X 1$ mRNA copy numbers mRNA; C. The relative TP53BP1 mRNA copy numbers. AL: Mammary gland tissues of mice fed Ad libitum chow; 0.5ED: $0.5 \mathrm{mg} / \mathrm{kg}$ estradiol-treated mouse mammary gland tissues; 4ED: $4 \mathrm{mg} / \mathrm{kg}$ estradiol-treated mouse mammary gland tissues. ${ }^{*} p<0.05 ;{ }^{* *} p<0.01 ; * * * * p<0.0001$ (NC: $n=9$; $0.5 \mathrm{E}: n=3 ; 4 \mathrm{E}: n=9)$.

Accumulating evidence shows that the incidence of breast cancer among women in the industrialized countries is most probably related to the environmental contamination by estrogen-like chemicals ${ }^{[23]}$. Nevertheless, it has been unknown how can estrogens per se trigger breast cancer.From the carcinogenic mechanism, however, it has been clear that estradiol can produce 2-hydoxyestradiol and 4-hydoxyestradiol at first, and then produce semi-quinone and quinone, which can react with DNA to form depurinated nucleotides and convert A-T to GC (point mutation) ${ }^{[13]}$. Additionally, estrogens can also increase the tumorigenic risk by inducing the methylation of repetitive DNA sequences, such as LINE-1 and Alu family ${ }^{[24]}$.

\subsection{Induced expression of mouse mammary gland tumor marker genes}

By quantifying the expression levels of mammary tumor marker genes, we found that CS+BC, like LPS, can decline the levels of mammary myoepithelium marker CK5-6 $6^{[25]}$, but elevate the levels of mammary hyperplasia marker $\mathrm{Ki}-67^{[26]}$. As to the tumor metastasis marker E-cadherin ${ }^{[27]}$ and the tumor differentiation marker 
GCDFP-15 ${ }^{[28]}$, no distinguishable changes were noted, suggesting that mammary tumor has been just formed, but has not yet entered the stage of metastasis and differentiation (Figure 5).
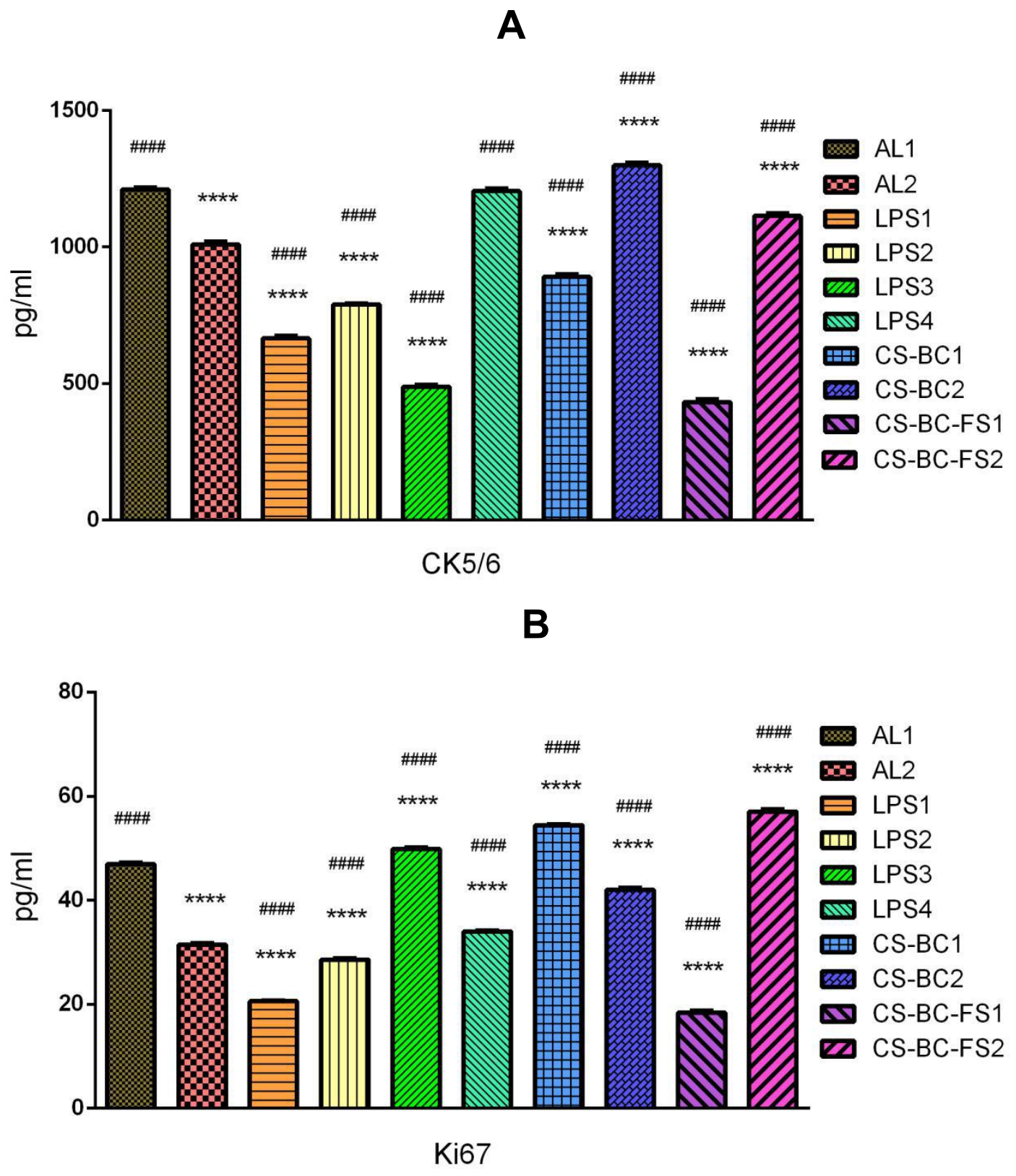

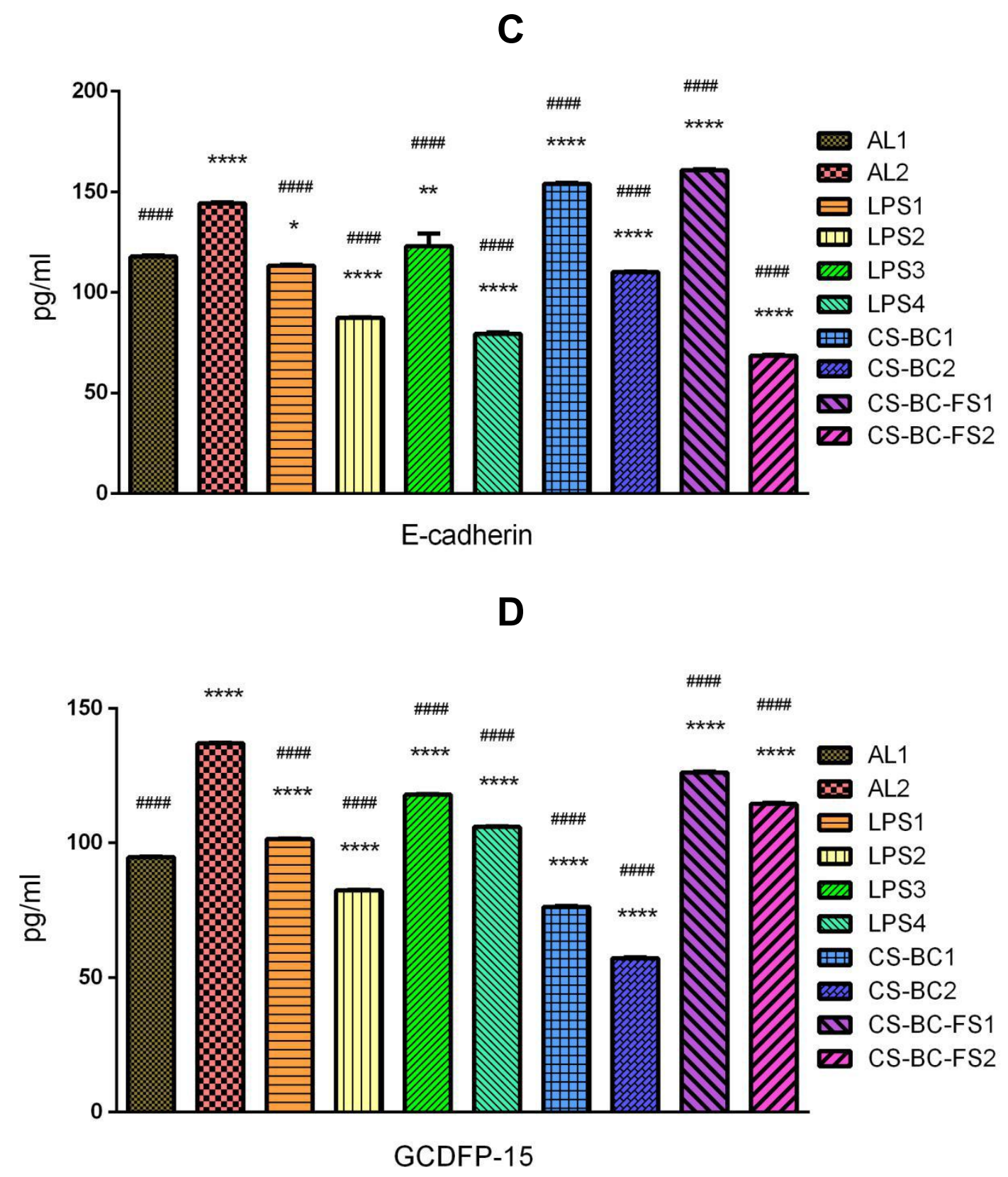

Fig. 5 The multi-factorial induction of tumor marker expression in mouse mammary gland tissues

A. CK5-6 levels; B. KI67 levels; C. E-cadherin levels; D. GCDFP-15 levels. AL: Mammary gland tissues of mice fed Ad libitum chow; LPS: LPS-treated mouse mammary gland tissues; CS+BC: chondroitin sulfate + Bacillus cereus-treated mouse mammary gland tissues; $\mathrm{CS}+\mathrm{BC}+\mathrm{FS}$ : chondroitin sulfate + Bacillus cereus + fulvestrant-treated mouse mammary gland tissues. * Compared to NC1; ${ }^{\#}$ Compared to NC. $p<0.05 ;{ }^{* *}$ or ${ }^{\# \#} p<0.01 ; * * * *$ or $^{\# \# \#} p<0.0001$ (NC: $n=6$; LPS: $n=12 ; \mathrm{CS}+\mathrm{BC}: n=6 ; \mathrm{CS}+\mathrm{BC}+\mathrm{FS}: n=6$ ).

From the above data, the individual variation remains a typical character, reflecting an affection from the individual gut microbiota variation. In similar, the expression levels of tumor marker genes are 
also individually variable, in which some are high expressed, while others are low expressed.

\subsection{Hypoxia-induced expression of angiogenesis genes}

To mimic the inflammatory inactivation of ERs, we employed the $\mathrm{ER}$ agonist $\mathrm{FS}$ to treat $\mathrm{CS}+\mathrm{BC}$ mice, and determined the serum $\mathrm{ED}$ level and hepatic HIF-1 $\alpha$ and VEGF levels. As results, it was shown that ED levels are higher in treated mice than those in control mice, addressing that FS can inhibit ER activity and complementarily increase ED levels. It was also observed that HIF-1 $\alpha$ levels are unchanged, whereas VEGF levels are dramatically elevated (Figure 6).

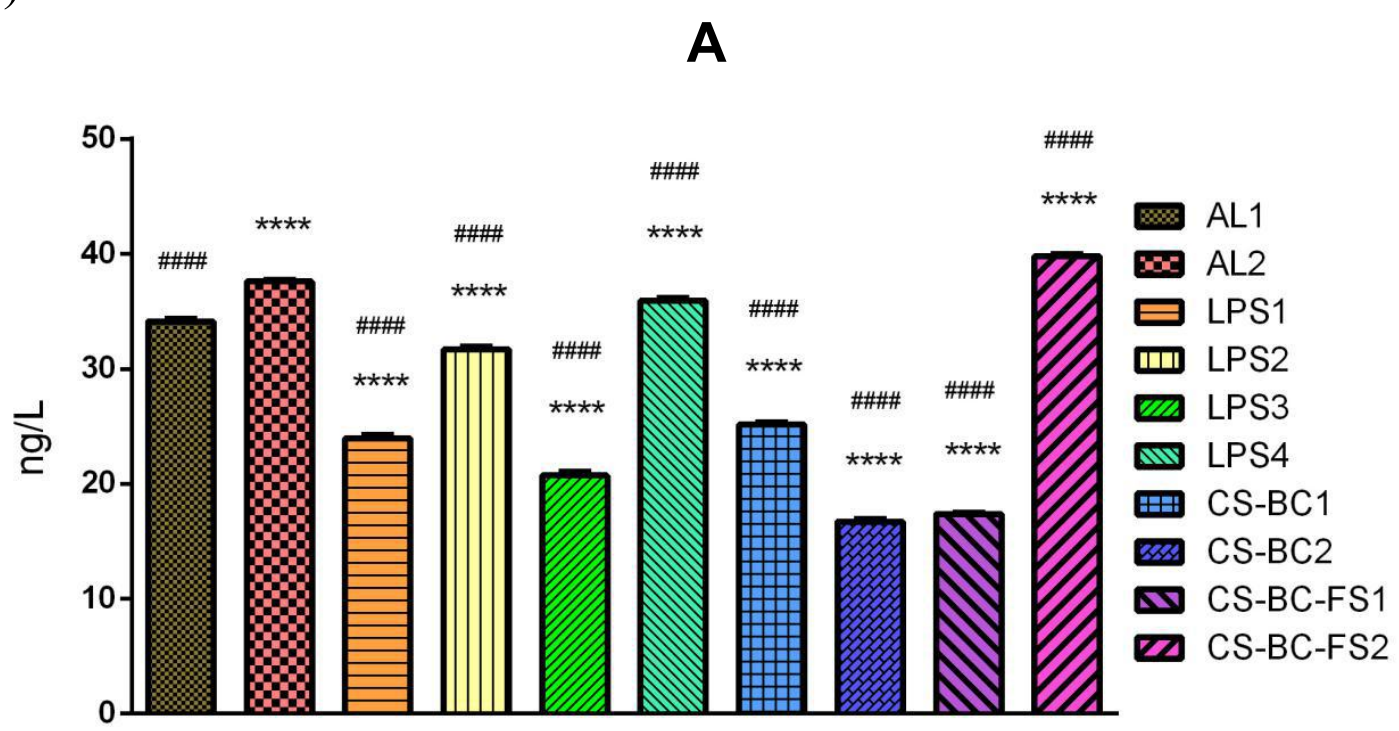

Estradiol



HIF-1a 


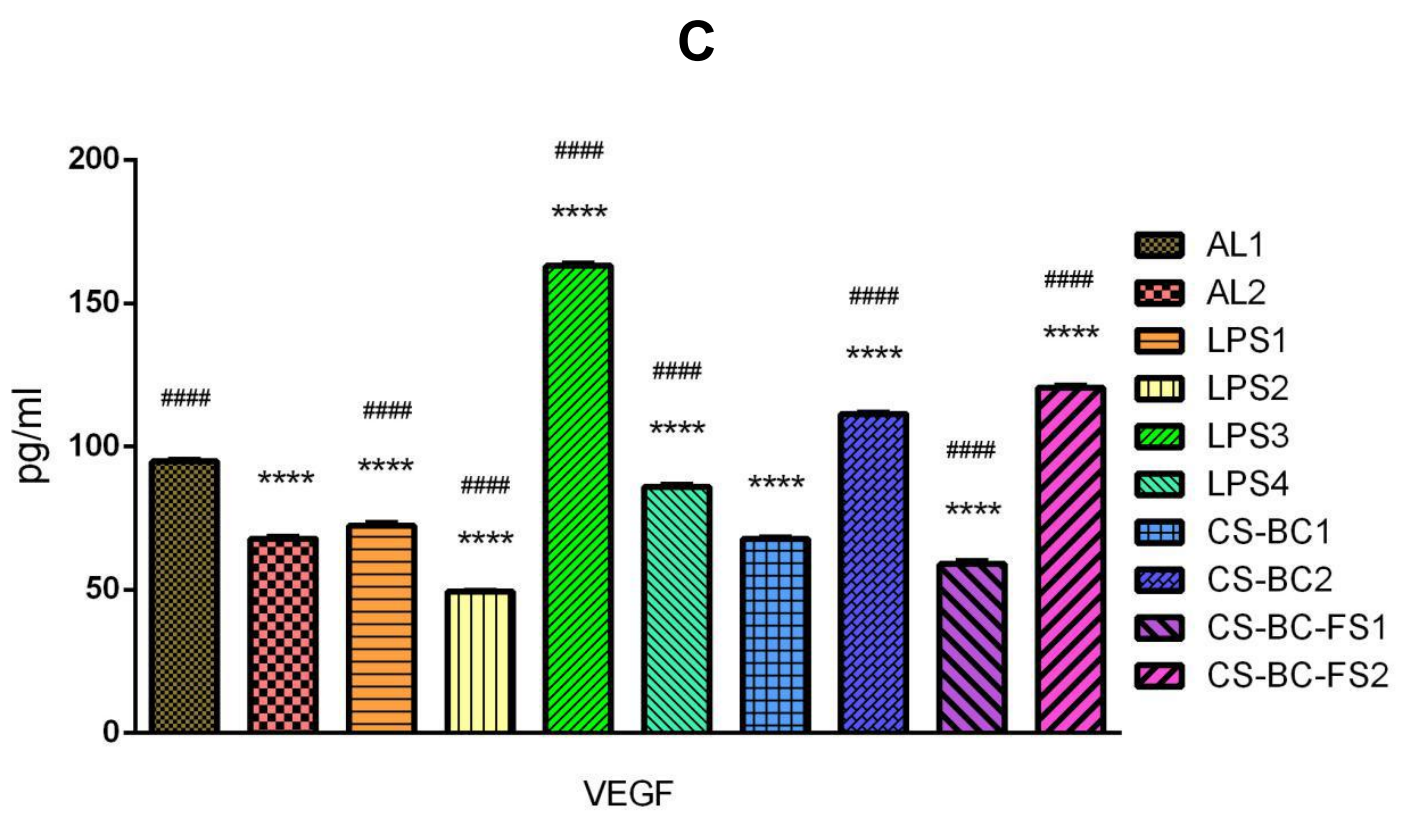

Fig. 6 The multi-factorial induction of mouse serum estradiol and liver HIF-1 $\alpha$ and VEGF levels

A. Estradiol levels; B. HIF-1 $\alpha$ levels; C. VEGF levels. AL: Serum or liver tissues of mice fed $A d$ libitum chow; LPS: LPS-treated mouse serum or liver tissues; CS+BC: chondroitin sulfate + Bacillus cereus-treated mouse mouse serum or liver tissues; $\mathrm{CS}+\mathrm{BC}+\mathrm{FS}$ : chondroitin sulfate+Bacillus cereus + fulvestrant-treated mouse mouse serum or liver tissues. ${ }^{*}$ Compared to NC1; ${ }^{\#}$ Compared to NC. $p<0.05 ; * *$ or $^{\# \#} p<0.01 ; * * * *$ or ${ }^{\# \# \#} p<0.0001$ (NC: $n=6$; LPS: $n=12$; $\mathrm{CS}+\mathrm{BC}: n=6 ; \mathrm{CS}+\mathrm{BC}+\mathrm{FS}: n=6)$.

It was known that the proinflammatory cytokine-activated hypoxia can induce VEGF expression and angiogenesis by HIF- $1 \alpha^{[29]}$. It was also evident that the expression of HIF- $1 \alpha$ is regulated at the post-translational level (activated upon translocation from the cytoplasm to the nuclei) rather than transcriptional and translational levels ${ }^{[30]}$.

\section{Conclusion}

The present study preliminarily testified the hypothesis of gut origin of breast cancer. By CS mimicking a meat diet, $\mathrm{BC}$ mimicking gut opportunistic infection, LPS mimicking hyper-endotoxinemia, ED mimicking hyperestrogenemia, and FS mimicking ER inflammatory inactivation, we obtained some evidence regarding the induced gene expression of mammary tumor-related transcription factors and tumor markers, and explained the tumor heterogeneity by the individual and tissue variations of gut dysbiosis and systemic 


\section{inflammation.}

Our next work should be re-acting a whole process of breast cancer at the cellular, tissue and individual levels, and eventually eradicating the risk of breast cancer by maintaining gut homeostasis.

\section{Acknowledgements}

\section{We thank Ms. Fan Pei in Novogene (Beijing, China) for her} assistance in meta-genomic analysis of the gut microbiome. This work was supported by the National Science Foundation of China (Grant No. 81273620; 81673861; 81774041).

\section{References}

1. McGuire A, Brown J A, Malone C, et al. Effects of age on the detection and management of breast cancer. Cancers, 2015, 7: 908-929

2. Wooster R, Weber B L. Breast and ovarian cancer. New Engl J Med, 2003, 348, 2339-2347

3. Yager J D, Davidson N E. Estrogen carcinogenesis in breast cancer. New Engl J Med, 2006, 354: $270-282$

4. Savage K I, Matchett K B, Barros E M, et al. BRCA1 deficiency exacerbates estrogen-induced DNA damage and genomic instability. Cancer Res, 2014, 74: 2773-2784

5. Kwa M, Plottel C S, Blaser M J, et al. The intestinal microbiome and estrogen receptor-positive female breast cancer. JNCI J Natl Cancer Inst, 2016, 108: djw029

6. Blackburn G L, Wang K A. Dietary fat reduction and breast cancer outcome: results from the Women's Intervention Nutrition Study (WINS). Am J Clin Nut, 2007, 86: s878-s881

7. Johnson K C, Miller A B, Collishaw N E, et al. Active smoking and secondhand smoke increase breast cancer risk: the report of the Canadian Expert Panel on Tobacco Smoke and Breast Cancer Risk (2009). Tobacco control, 2011, 20: e2

8. Boffetta $\mathrm{P}$, Hashibe $\mathrm{M}$, La Vecchia $\mathrm{C}$, et al. The burden of cancer attributable to alcohol drinking. Intern J Cancer, 2006, 119: 884-887

9. Yang J, Tan Q, Fu Q, et al. Gastrointestinal microbiome and breast cancer: correlations, mechanisms and potential clinical implications. Breast Cancer, 2016, 24: 220-228

10. Li C Q, Zheng Q, Wang Q, et al. Biotic/abiotic stress-driven Alzheimer's disease. Front Cell Neurosci, 2016, 10: 269

11. Rey F E, Gonzalez M D, Cheng J, et al. Metabolic niche of a prominent sulfate-reducing human gut bacterium. Proc Natl Acad Sci USA, 2013, 110: 13582-13587

12. Desai MS, et al. Dietary fiber-deprived gut microbiota degrades colonic mucus barriers and enhances pathogen susceptibility. Cell, 2016, 167:1339-1657

13. Mailander P C, Meza J L, Higginbotham S, et al. Induction of A.T to G.C mutations by erroneous repair of depurinated DNA following estrogen treatment of the mammary gland of ACI rats. J Steroid Biochem Mol Biol, 2006, 101: 204-215

14. Lai A C, Crews C M. Induced protein degradation: an emerging drug discovery paradigm. Nat Rev Drug Discov, 2017, 16: 101-114 
15. Qin J, et al. A metagenome-wide association study of gut microbiota in type 2 diabetes. Nature, 2012, 490: 55-60

16. Shang Q, Shi J, Song G, et al. Structural modulation of gut microbiota by chondroitin sulfate and its oligosaccharide. Int J Biol Micromol, 2016, 89: 489-498

17. Desai MS, Seekatz AM, Koropatkin NM, et al. A dietary fiber-deprived gut microbiota degrades the colonic mucus barrier and enhances pathogen susceptibility. Cell, 2016, 167 : 1339-1353.

18. Shang Q, Yin Y, Zhu L, et al. Degradation of chondroitin sulfate by the gut microbiota of Chinese individuals. Int J Biol Micromol, 2016, 86: 112-118

19. Hersoug LG, Moller P, Loft S. Gut microbiota-derived lipopolysaccharide uptake and trafficking to adipose tissue: implication for inflammation and obesity. Obes Rev, 2016, 17: 297-312

20. Khale WT, Lee SC, Stingl J, et al. BCL11A is a triple-negative breast cancer gene with critical functions in stem and progenitor cells. Nat Commun, 2015, 6: 5987

21. Ferrari N, Mohammed ZMA, Nixon C, et al. Expression of RUNX1 correlates with poor patient prognosis in triple negative breast cancer. PLoS One, 2014, 9: e100759

22. Bouwman P, Aly A, Escandell JM, et al. 53BP1 loss rescues BRCA1 deficiency and is associated with triple-negative and BRCA-mutated breast cancers. Nat Struct Mol Biol, 2010, 17:688-695

23. Lecomte S, Habauzit D, Charlier TD, et al. Emerging estrogenic pollutants in the aquatic environment and breast cancer. Genes (Basel), 2017, 8: pii: E229

24. Boyne DJ, Friedenreich CM, McIntyre JB, et al. Endogenous sex hormone exposure and repetitive element DNA methylation in healthy postmenopausal women. Cancer Causes Control, 2017, doi: 10.1007/s10552-017-0958-z

25. Maeda T, Nakanishi Y, Hirotani Y, et al. Immunohistochemical co-expression status of cytokeratin 5/6, androgen receptor, and p53 as prognostic factors of adjuvant chemotherapy for triple negative breast cancer. Med Mol Morphol, 2016, 49:1 1-21

26. Borges US, Costa-Silva DR, da Silva-Sampaio JP, et al. A comparative study of Ki-67 antigen expression between luminal A and triple-negative subtypes of breast cancer. Med Oncol, 2017, 34:156

27. Tan R, Wang L, Song J, et al. Expression and significance of Twist, estrogen receptor, and E-cadherin in human breast cancer cells and tissues. J Cancer Res Ther, 2017, 13: 707-714

28. Darb-Esfahani S, von Minckwitz G, Denkert C, et al. Gross cystic disease fluid protein 15 (GCDFP-15) expression in breast cancer subtypes. BMC Cancer, 2014, 14: 546

29. Bao F, Wu P, Xiao N, et al. Nitric oxide-driven hypoxia initiates synovial angiogenesis, hyperplasia and inflammatory lesions in mice. PLoS One, 2012, 7: e34494

30. van Uden P, Kenneth NS, Rocha S. Regulation of hypoxia-inducible factor-1alpha by NF-kappaB. Biochem J, 2008, 412: 477-484 